\title{
Publizistenpreis der deutschen Bibliotheken 2019 geht an Susanne Brahms
}

\author{
Preisverleihung am 21.03.2019 auf dem Bibliothekskongress in \\ Leipzig
}

http://doi.org/10.1515/bd-2019-0049

Der mit 7.500 Euro dotierte Publizistenpreis der deutschen Bibliotheken (HelmutSontag-Preis) geht in diesem Jahr an die freie Autorin Susanne Brahms. Ausgezeichnet wird ihre dokumentarische Fernseharbeit „Die Bücherjäger. Kampf um das Wissen der Welt“ (ARTE, 2017).

Die Preisverleihung durch den Deutschen Bibliotheksverband (dbv), den Berufsverband Bibliothek Information e.V. (BIB) und den Verein Deutscher Bibliothekarinnen und Bibliothekare e.V. (VDB) fand am 21. März 2019 im Rahmen der Abschlussveranstaltung des Bibliothekskongresses in Leipzig statt. Die Einreichung von Susanne Brahms wurde von einer unabhängigen Jury, bestehend aus Vertreter^innen der bibliothekarischen Verbände (BIB, dbv und VDB) sowie von Medien und Verlagen, ausgewählt.

In der der Jurybegründung heißt es:

„In dem Arte-Beitrag ,Bücherjäger‘ vom 17.10.2017 begleitet die freie Autorin Susanne Brahms den Benediktinermönch Columba Stewart aus Minnesota, USA, der seit über 30 Jahren durch die Krisenregionen der Welt reist. Er sucht alte Manuskripte, zum Beispiel im Irak, wo es ihm gelungen ist, eine uralte Klosterbibliothek digitalisieren zu lassen. Im Irak unterstützt er auch den Dominikanermönch Najeeb Michael, der seine Bibliothek bereits zweimal unter dramatischen Umständen vor dem Zugriff radikaler Islamisten gerettet hat.

Susanne Brahms zeigt mit ihrem Beitrag anschaulich und spannend, wie diese modernen Bücherjäger auch im 20. Jahrhundert Kulturgut retten. In der Vielvölkerstadt Sarajevo bestand beispielsweise eines der Kriegsziele in den 1990er Jahren darin, die Erinnerung an das Zusammenleben der Ethnien auszulöschen: Die Armee der bosnischen Serben schoss deshalb die Nationalbibliothek und das Orient-Institut in Brand. Susanne Brahms besuchte für ihren Film Mustafa Jahic, der damals Bibliothekar der letzten Handschriftensammlung Sarajevos war und 20.000 Manuskripte aus dem Mittelalter in Bananenkartons rettete, die er achtmal unter Lebensgefahr durch die eingekesselte Stadt trug.

Der Film ,Bücherjäger' ist eine behutsame Dokumentation über die historische Funktion von Bibliotheken, die heute, vor dem Hintergrund von Krieg und 
Zerstörung, kaum aktueller sein könnte: Das Wiederherstellen von Handschriften in mühevoller Kleinstarbeit und unter schwierigsten Bedingungen, die Digitalisierung und das Bewahren von kulturellem Erbe werden in dem Film sehr persönlich anhand der Figuren erzählt. Damit ist es Susanne Brahms nach Auffassung der Jury gelungen, einer breiten Fernsehöffentlichkeit die Bedeutung von Wissenszugänglichkeit und dem Bewahren von kulturellem Gedächtnis zu eröffnen.“

Der Preis würdigt Publizisten, die das Bibliothekswesen durch herausragende Einzelbeiträge oder durch die Kontinuität sachgerechter Berichterstattung wirkungsvoll gefördert haben. Er wird seit 1987 jährlich verliehen. Die Initiative zur Preisverleihung geht auf die Anregung des ehemaligen dbv-Vorsitzenden Helmut Sontag (1934-1988) zurück. Von 2010 bis 2017 erfolgte die Ausschreibung gemeinsam mit der Wissenschaftlichen Buchgesellschaft (WBG). Seit 2018 schreiben die bibliothekarischen Verbände Berufsverband Bibliothek Information e.V. (BIB), der Verein Deutscher Bibliothekarinnen und Bibliothekare e.V. (VDB) und der Deutsche Bibliotheksverband e.V. (dbv) den Publizistenpreis der deutschen Bibliotheken gemeinsam aus.

Weiter Informationen im Internet:

- http://www.bibliotheksverband.de/dbv/auszeichnungen/publizistenpreis. html (Zum Preis)

- http://www.bibliotheksverband.de/dbv/auszeichnungen/publizistenpreis/ preistraeger (Zur Preisträgerin)

\section{Kontakt:}

Deutscher Bibliotheksverband e.V. (dbv)

Maiken Hagemeister, Pressesprecherin und Leitung Kommunikation, Tel.: 0 30/644 9899 25, E-Mail: hagemeister@bibliotheksverband.de, http://www.bibliotheksverband.de, http://www.bibliotheksportal.de 